\title{
Antiphospholipid syndrome
}

This is the last in a series of occasional articles by patients about their experiences that offer lessons to doctors. For more information about our current series, "What Your Patient is Thinking," contact Rosamund Snow, patient editor, rsnow@bmj.com

\section{Tracy Jallow patient living with antiphospholipid syndrome ${ }^{1}$, David D'Cruz professor ${ }^{2}$, Heidi Lempp senior lecturer in medical sociology ${ }^{3}$}

${ }^{1}$ Sydenham, London; ${ }^{2}$ Louise Coote Lupus Unit, Guy's and St Thomas' Hospital, London, UK ; ${ }^{3}$ King's College London, Faculty of Life Sciences and Medicine, Academic Rheumatology, Clinical Trials Group, UK

\section{The jigsaw pieces}

Imagine a box of jigsaw pieces tipped out on the floor, all the bits are there, but to have a clear picture, I first had to put them all together to make sense. This was a journey that I was to take.

I had a normal childhood. One delay that stands out was that I did not begin menstruating until the age of 18 , and my periods were irregular. Around this time I experienced stabbing stomach pains that left me short of breath. My two pregnancies in 1990 and 1997 were complicated because I experienced severe unexplained abdominal pain, vomiting, and fatigue during and after the birth, and I put my forgetfulness (not remembering friends' names) down to becoming a mum.

After the birth of my second child I started vomiting every day. After two weeks I was admitted to hospital and after another few weeks of investigations I was taken to theatre as an emergency. A computed tomography scan showed a "swelling" in my abdomen. I was diagnosed with an unexplained blood clot in my large bowel that had burst. The incident was explained to me as "a mystery" as I had had a normal second birth.

After discharge following my bowel operation I was promptly readmitted with a suspected pulmonary embolism. I was treated with heparin injections, and a computed tomography scan was inconclusive. In 2001, I returned from a holiday abroad with a throbbing pain in my left leg. I had several blood tests, but was not contacted about the results by the hospital staff. The pounding pain subsided and I forgot all about the discomfort. When I finally got a diagnosis of antiphospholipid syndrome I requested access to my GP records to look for clues or things that had been missed; looking back through my notes I saw that a lupus anticoagulant blood test was positive.

In the following years I underwent several operations-for example, removal of my gall bladder and a thrombus in the portal vein. Both were accompanied by continuous extreme fatigue and "brain fog." I overheard a consultant commenting after one of the operations that my blood had been "acting weirdly," but I did not take any notice or ask why.

\section{Putting the pieces of the jigsaw together}

In 2008 it seemed to me that a guardian angel pointed me in the right direction. My left leg was constantly hurting, so my general practitioner referred me to a vascular consultant. He arranged a battery of blood tests and a Doppler examination of my left leg, which identified a clotting abnormality and showed that I had developed a blood clot in my popliteal artery and long saphenous vein. In addition, my kidney function was severely compromised so he urgently transferred me to a haematologist. Apart from the ongoing fatigue and pain I now had a continuous headache. The haematologist took a detailed history, advised me not to travel by plane, but instead ordered specific blood tests, a magnetic resonance imaging scan, and an angiogram. I think now that this consultant saved my life. I felt physically and emotionally exhausted. To my surprise the scan and angiogram showed that my right kidney and coeliac and superior mesenteric arteries were occluded. A subsequent brain scan showed high signal lesions. A week later I was referred to the lupus clinic where I was diagnosed with a rare autoimmune condition called primary vascular antiphospholipid syndrome, which causes an increased risk of blood clots (and miscarriages). My medical history now suddenly all made sense to me and I finally had a name to explain my strange, unexplained symptoms over so many years.

\section{Life after diagnosis}

The first few months after diagnosis were hectic. I had to get my drug regimen stabilised. The treatment was lifelong anticoagulation that required close regular blood monitoring. I 
was also diagnosed as having stage 3 kidney failure and therefore my hypertension required frequent check-ups (I had lost one kidney already). I was prescribed hydroxychloroquine for muscle aches in my arms and legs, and fatigue.

I continue to work full time in "planning and performance" for a government department. My employers have been sympathetic and understanding about my illness and have made reasonable adjustments for me. I describe my body like an old car-I cannot sit in it and drive off. Instead, when I wake up in the morning I have to gently start myself up before I am able to function physically and mentally-sometimes I cannot function at all. Every day I experience antiphospholipid syndrome in a different way.

\section{Personal reflection and self management}

Unfortunately, it took 17 years to receive a diagnosis, which meant a lot of damage was already done to my body. I was introduced to the manager of the Hughes Syndrome Foundation, who provided me with leaflets and important information about antiphospholipid syndrome. Going forward is the only way for me and I have to make the best of my situation. I have therefore decided to raise awareness and educate people about antiphospholipid syndrome, including family, friends, and colleagues, and sometimes even doctors. Many healthcare professionals that I meet have not come across patients with antiphospholipid syndrome and therefore lack information about this rare autoimmune condition. I have learnt not to ignore symptoms. I have decided that it is better to consult a doctor to avoid further negative consequences to my health or miss out on vital treatment.

I manage my pain through painkillers and heat therapy-hot water bottles, hot baths, and saunas. Some of the pain, I have discovered, seems to be triggered by specific foods-for example, wheat, red meat, and certain fruits and vegetables. I therefore follow a diet that facilitates easy digestion such as fish, chicken, rice, and bananas. I still have stomach pains on a daily basis. I use a walking stick most of the time because of leg pain and joint stiffness. This visual aid lets people know that I am unwell as antiphospholipid syndrome is mostly invisible to others. I had to learn to pace myself-if I do not rest during the day, I experience unnecessary pain and fatigue and I am mentally unfocused. I have come to accept that I have a lifelong condition and that it is not going away, and to be honest it has contributed to who I am now.

\section{Lessons learnt over the years}

Since my diagnosis I no longer ignore any symptoms that I experience and make sure that I bring them to the attention of my GP or consultant. I also ask more questions as I believe my own understanding of my condition plays a vital part in my wellbeing and helps me to stay in control. I prefer to be treated as a whole person, instead of attention being paid to my coagulation time (international normalised ratio) only. I also like to ask medical and nursing staff questions so that I can untangle in my mind whether new symptoms are pieces of the complex antiphospholipid syndrome jigsaw puzzle or something else.

Competing interests: We have read and understood BMJ policy on declaration of interests and declare the following. DDC is a trustee of the Hughes Syndrome Foundation. HL has received funding over the last 36 months from the following funders as a principal investigator or co-applicant: National Institutes of Health Research, Arthritis Research UK, South London Membership Council, Guy's and St Thomas' Charity, London; European Union, Health Foundation.

Provenance and peer review: Not commissioned; not externally peer reviewed.

Cite this as: BMJ 2015;350:h1426

๑ BMJ Publishing Group Ltd 2015 


\section{Useful information}

The Hughes Syndrome Foundation (www.hughes-syndrome.org) is the only UK registered charity dedicated to helping people affected by antiphospholipid syndrome. Its mission is to help save and improve the lives of patients with the syndrome by achieving early diagnosis and the best possible treatment. It does this by

- Informing doctors, nurses, and GPs about how to look for, test, and treat antiphospholipid syndrome

- Raising awareness in the media and general public about the condition

- Offering support and understanding to anyone affected by the illness

- Raising funds to provide support, education, and research

\section{APS information sites}

NHS Choices: www.nhs.uk/conditions/hughes-syndrome/pages/introduction.aspx

Patient UK: www.patient.co.uk/doctor/Antiphospholipid-syndrome.htm

\section{A doctor's perspective}

Antiphospholipid syndrome is characterised by arterial and venous thrombosis and morbidity during pregnancy. Diagnosis is made on clinical grounds and is supported by positive tests for antibodies to phospholipid binding proteins including anticardiolipin antibodies and the lupus anticoagulant. Anti- $\beta_{2}$-glycoprotein 1 antibodies add little diagnostically if anticardiolipin antibodies or lupus anticoagulant or both are positive, but can provide useful prognostic information if all three autoantibodies are present. Patients with all three types of autoantibody are thought to be at high risk of thrombotic events.

Tracy's story illustrates the often long delays experienced by patients before a diagnosis of antiphospholipid syndrome is made and treatment started. In Tracy's case the presenting features of severe abdominal and loin pain were unusual, and in retrospect were almost certainly attributable to infarction of the right kidney. Subsequent symptoms of abdominal pain on eating suggested mesenteric angina and led to imaging studies that identified occlusion of the coeliac and superior mesenteric arteries. Her leg pains and claudication symptoms were caused by bilateral popliteal artery occlusions, although fortunately she did not experience critical limb ischaemia which has, in other patients, led to amputation. Her renal biopsy and renal imaging confirmed classic antiphospholipid syndrome nephropathy with thrombotic microangiopathy and focal cortical infarction, which was associated with severe hypertension. Multiple venous events included portal vein and long saphenous vein thrombosis and pulmonary embolism. Cognitive dysfunction, which Tracy described as brain fog, forgetfulness, and headaches, is common in these patients, and the causes are poorly understood. Brain magnetic resonance imaging often shows so called high signal lesions but may also show multiple lacunar cerebral infarcts. In Tracy's case the lesions were reported as non-specific foci of T2 hyperintensity in keeping with small vessel disease rather than lacunar infarcts.

Although her two pregnancies were complicated by abdominal pain and vomiting, Tracy did not experience pregnancy morbidity, which includes spontaneous pregnancy loss, fetal death, intrauterine growth restriction, pregnancy induced hypertension, pre-eclampsia, and stillbirth. In patients with obstetric antiphospholipid syndrome, pre-pregnancy counselling is crucial and treatment with aspirin and low molecular weight heparin has transformed the outcome of these often high risk pregnancies. Patients should continue to take heparin and aspirin in the postpartum period.

Tracy's quality of life has been profoundly affected by antiphospholipid syndrome. In addition to the potentially life and organ threatening thrombotic events, the other aspects of the disease such as cognitive dysfunction and fatigue have been debilitating. These remain poorly understood not just in this syndrome, but in many other autoimmune rheumatic disorders such as systemic lupus erythematosus.

Patients like Tracy who have experienced multiple arterial and venous events should be treated with long term oral anticoagulants such as warfarin, with a high target international normalised ratio of 3.0 to 4.0. Management of other cardiovascular risk factors is also vital to reduce the risk of further events. Tracy's most important risk factor was hypertension, which required three agents to achieve good control. About a third of these patients have lupus-like symptoms such as arthralgia (in the absence of typical lupus serology), and these symptoms respond to hydroxychloroquine. This agent has the added advantage of reducing thrombotic risk in conjunction with warfarin and is generally well tolerated as a long term treatment.

David D'Cruz, professor david.d'cruz@kcl.ac.uk

Heidi Lempp, senior lecturer in medical sociology 